
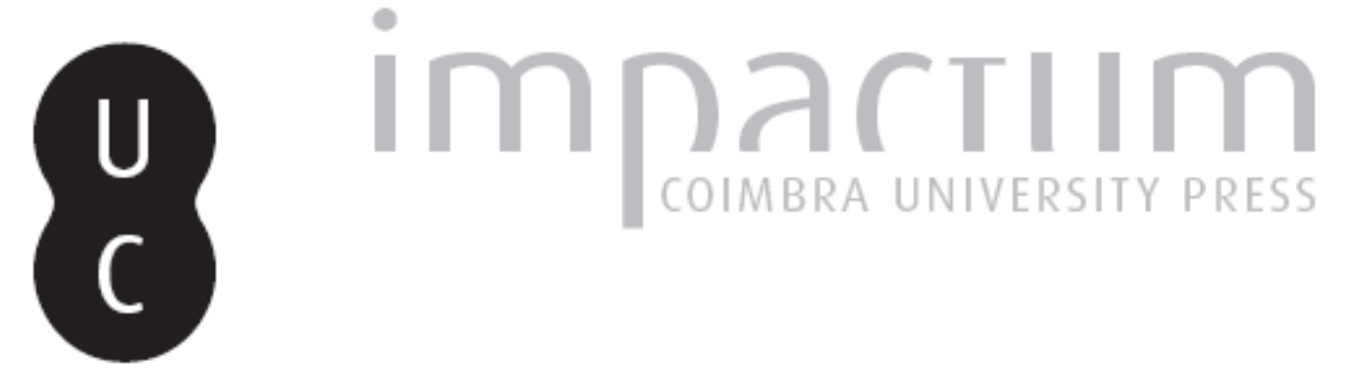

Due pittori e due cardinali: una riflessione su Scipione e Candido Dominguez Alvarez Autor(es): $\quad$ Rosa, Stefano De

Publicado por: Imprensa da Universidade de Coimbra

URL persistente: URl:http://hdl.handle.net/10316.2/42451

DOI: $\quad$ DOI:https://doi.org/10.14195/0870-8584_0_8

Accessed : $\quad$ 26-Apr-2023 08:16:17

A navegação consulta e descarregamento dos títulos inseridos nas Bibliotecas Digitais UC Digitalis, UC Pombalina e UC Impactum, pressupõem a aceitação plena e sem reservas dos Termos e Condições de Uso destas Bibliotecas Digitais, disponíveis em https://digitalis.uc.pt/pt-pt/termos.

Conforme exposto nos referidos Termos e Condições de Uso, o descarregamento de títulos de acesso restrito requer uma licença válida de autorização devendo o utilizador aceder ao(s) documento(s) a partir de um endereço de IP da instituição detentora da supramencionada licença.

Ao utilizador é apenas permitido o descarregamento para uso pessoal, pelo que o emprego do(s) título(s) descarregado(s) para outro fim, designadamente comercial, carece de autorização do respetivo autor ou editor da obra.

Na medida em que todas as obras da UC Digitalis se encontram protegidas pelo Código do Direito de Autor e Direitos Conexos e demais legislação aplicável, toda a cópia, parcial ou total, deste documento, nos casos em que é legalmente admitida, deverá conter ou fazer-se acompanhar por este aviso. 


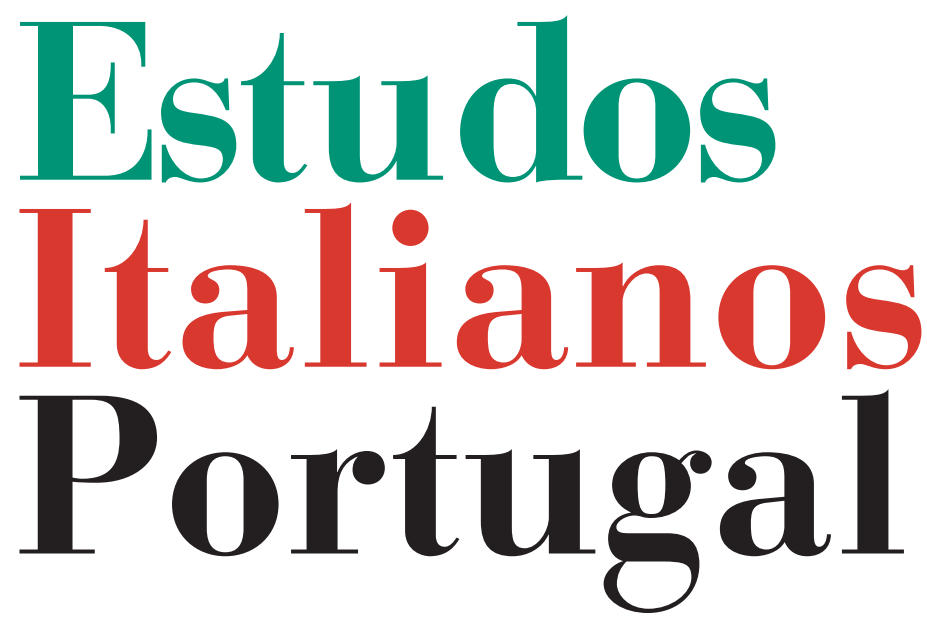

Instituto

Italiano

de Cultura

de Lisboa

Nova Série

$\mathrm{N}^{\mathbf{0}} \mathbf{0}$ 


\section{DUE PITTORI E DUE CARDINALI. \\ UNA RIFLESSIONE SU SCIPIONE \\ E CANDIDO DOMINGUEZ ALVAREZ}

Stefano De Rosa

Alla Biennale di Venezia del 1930, fece scalpore un quadro, Ritratto del Cardinale Decano (Il Cardinale Vannutelli), di Gino Bonichi, che si firmava Scipione ${ }^{1}$. La Giuria d'ammissione ebbe più di un motivo d'inquietudine, di fronte a quel Cardinale sofferente, la cui epidermide pare sul punto di sbriciolarsi, rosa all'interno da un processo di corruzione.

Il dipinto era quanto di più lontano si potesse immaginare dalla Chiesa trionfante che una certa arte di regime stava allora celebrando. Nel dipingere il Cardinale, Scipione non aveva avuto nessuna intenzione polemica. Giovane minato da un male incurabile, il pittore sapeva leggere negli altri i presagi della fine. Scavava le fisionomie con un'asciutta profondità d'introspezione, che non cedeva alla retorica.

Nel dipinto, Scipione assorbì il fascino di una tradizione pittorica che lo riconnetteva al Paolo III con i nipoti di Tiziano e all'Innocenzo $X^{o}$ di Velasquez. Quel dipinto l'aveva ossessionato per mesi. In esso, Scipione vedeva una tensione espressiva che forza i tratti della linea, impiegata per rubare le verità più nascoste degli occhi e del volto. In Velasquez,

1 XVII Biennale di Venezia, Sala 28, n. 24. Sull'artista, nato a Macerata nel 1904 e precocemente scomparso ad Arco nel 1933, esiste una letteratura critica abbastanza folta. Per un'impeccabile ricostruzione biografica, un efficace bilancio critico e per una bibliografia ben documentata, mi limito a segnalare Scipione. Vita e Opere, di Maurizio Fagiolo dell'Arco e Valerio Rivosecchi, Torino, Umberto Allemandi Editore, 1998. Sul dipinto, pp. 107-109; 306-307. 
inoltre, Scipione trovava un esempio inarrivabile di un'arte che trattiene un valore taumaturgico, guarisce dalle pene mostrandole, senza ammantarle con veli di estetismi ipocriti.

Il modello di Scipione, il novantaquattrenne Cardinale Vannutelli, aveva fama di essere il vero motore del Vaticano ${ }^{2}$. Uomo astutissimo, capace di ascoltare a lungo e di concludere le udienze con frasi di straordinaria efficacia, il Cardinale esercitava una profonda influenza su Scipione. Il pittore si sentiva attratto dal cattolicesimo seicentesco, di cui avvertiva i rovelli spirituali e i rigori dottrinari, non meno delle aperture filosofiche e scientifiche che quel tempo dispensava all'umanità. La fede di Scipione era sotto il segno della contraddittorietà e della tragedia. Il Cardinale Vannutelli era la persona che incarnava il tormentato rapporto dell'artista con la fede e con la romanità. Come ha testimoniato il poeta Leonardo Sinisgalli, Scipione s'attaccò ferocemente al suo modello, non lo perse mai di vista, "lo guardò, lo descrisse, con le sue avide narici Scipione lo fiutò, fiutò il suo odore, l'odore di santità sotto la porpora, sotto l'ermellino, assisté infine alla sua decomposizione sul letto di morte."3

Il quadro di Scipione, un olio su tavola di notevoli dimensioni $^{4}$, reca sul retro un altro abbozzo, a pennello, per la

2 Si veda, in proposito, il necrologio pubblicato su Il Giornale d'Italia del 10 luglio 1930, nel quale si scriveva che il Cardinale era un uomo per il quale non erano "mai esistiti momenti di debolezza."

3 Secondo Sinisgalli, Scipione "aveva guardato il ritratto del Cardinale di Velazquez alla Galleria Doria Pamphili: i grandi preti, i principi della chiesa dal colorito paonazzo, doveva considerarli dei purosangue, stracchi, nutriti di latino e di cibi prelibati, vecchi dalle cartilagini aride, con qualcosa sulla pelle che ha del pollastro, un orgoglio nelle vesti e nel sangue la ferocia dei tacchini, i tristi uccelli armati, le sentinelle dell'Ade". Il testo di Leonardo Sinisgalli fu pubblicato su Aretusa, Roma, settembre 1945, ed é riproposto in Scipione. Vita e Opere, cit., pp. 306-307.

${ }^{4} \mathrm{Cm} .133,7 x 117,3$. Per avvicinarsi alla definitiva versione del ritratto, Scipione eseguì disegni e bozzetti, uno dei quali é conservato a Firenze; cfr. Raccolta d'Arte Contemporanea Alberto Della Ragione, a cura di Fiorenza Scalia, Firenze, Edizioni Centro Di, 1987, n. 45 (olio su tavola, cm. 53×43). 
composizione di un testo che appare fra i più studiati del pittore. Tiziano e Velasquez lo incalzavano da vicino, e Scipione non voleva perdere il confronto con i suoi modelli prediletti.

Il quadro fu ammesso alla Biennale solo per volontà di Cipriano Efisio Oppo, membro della Giuria, che per esso si batté con generosità. Ebbe l'attenzione critica di Lionello Venturi e di altri storici e critici d'arte ${ }^{5}$. Il Cardinale era da poco scomparso, quando il quadro fu esposto. Gli eredi non lo vollero, e anzi si adoprarono affinché il dipinto, da loro considerato irriverente verso il potente congiunto, andasse perduto $^{6}$. Fu pubblicato sul catalogo della Biennale.

O Bispo di Candido Dominguez Alvarez (1906-1942) è un dipinto che ha molte analogie con il Ritratto del Cardinale Decano di Scipione. Il quadro del pittore portoghese è del 1933. "Alucinado vencido da vida", Dominguez Alvarez iniziò a dipingere intorno al 19247. Nato a Oporto da una famiglia di origine gallega, Dominguez Alvarez aveva seguito un corso per diventare funzionario delle poste. Si era impiegato, ma non era soddisfatto della sua vita. Nel 1926 s'iscrisse all'Accademia di Belle Arti di Oporto, ed iniziò a frequentare i corsi di architettura. L'anno successivo abbandonò la classe di architettura, per iscriversi a pittura. Le sue prime prove pittoriche sono dei paesaggi urbani desolanti, nei quali il processo d'industrializzazione mostra il prezzo imposto alla sua città, che comincia a perdere

${ }^{5}$ L. Venturi, in Belvedere, Milano, n. 5-6, Maggio-Giugno 1930; U. Nebbia, XVII Esposizione Biennale d'Arte, Venezia-Milano, 1930 e "Emporium”, Bergamo, Giugno 1930; V. Costantini, in L'Italia Letteraria, Roma, Maggio 1930.

${ }^{6}$ Dopo alcuni passaggi, il quadro é stato acquistato dalla Galleria Comunale d'Arte Moderna di Roma.

${ }^{7}$ La letteratura critica sull'artista è fortunatamente ampia. Mi limito qui a ricordare il catalogo della mostra di Lisbona, Galleria Almada Negreiros, 2-30 aprile 1987, a cura di Isabel Oliveira e Silva, e il più recente catalogo della mostra tenutasi a Oporto, Casa de Serralves, luglio 1987, con testi di Isabel Oliveira e Silva e Alberto de Serpa. 
quell'aspetto di dolce dedalo di strade brevi e contorte, che l'aveva resa famosa.

Nel 1929 Candido Dominguez Alvarez si presentò in una mostra collettiva di allievi dell'Accademia di Oporto. La mostra era un omaggio al pittore Marques de Oliveira, pittore naturalista, che nel suo insegnamento indicava la necessità di rompere con gli schemi accademici. Intorno a Marques de Oliveira si raccolse un gruppo di giovani, per lo più studenti, che si presentarono con un manifesto ribollente di ribellione e di rabbia generazionale contro il mondo dell'Accademia. Il gruppo si chiamava Mais Além. Teorizzava il dovere dell'impegno sociale dell'artista, che doveva cercare la verità e respingere le tentazioni dello stile.

Dominguez Alvarez uscì dal gruppo quello stesso anno. Un periodo di vacanza trascorso in Galizia, gli permise di vedere riproduzioni di quadri cubisti e astratti, facendogli comprendere il ritardo culturale accumulato dal Portogallo settentrionale. Lisbona, nel 1929, aveva già conosciuto la provocazione del Futurismo, ed aveva dato vita all'intensa ed esaltante stagione del Modernismo ${ }^{8}$. Prima di finire ingabbiato dal regime fascista di Salazar e dall'opportunismo politico di Antonio Ferro, il Modernismo aveva vissuto una fase eroica, illuminata dalla pittura di José de Almada Negreiros, dalle sperimentazioni di Amadeo de Souza-Cardoso, dalla cultura aggiornata di Viana, e sorretto sempre da una critica militante colta e attenta, nelle file della quale si segnalava Pessoa ${ }^{9}$. Poco di tutto questo, era giunto a Oporto.

${ }^{8}$ Per un'efficace sintesi sul Modernismo in Portogallo, si veda J.-A. França, O Modemismo na arte portuguesa, Lisboa, Ministério da Educação, 1991.

9 Su Amadeo de Souza Cardoso si vedano il Jornal da Exposição edito dalla Fondazione Gulbenkian di Lisbona, agosto 1987, e il catalogo della mostra svoltasi a Bruxelles, Musée d'Art Moderne, 27 luglio-8 dicembre 1991, con testi di Margarida Acciaiuoli, Pierre Cabanne, Emidio Rosa de Oliveira, José-Augusto França, Ana Isabel Melo Ribeiro. Su José de Almada Negreiros esiste una vasta bibliografia. Mi sembra importante il taglio critico del Jornal da Exposição del 
Candido Dominguez Alvarez si rendeva conto di questa realtà, ma scelse di non spingersi verso sud, preferendo rimanere a combattere una guerra privata contro il mondo dell'arte. Dalla provincia portoghese nella quale si era volontariamente esiliato, Dominguez Alvarez uscì poche volte.

Espose 45 quadri nell'Ateneo Commerciale di Oporto nell'ambito di una mostra organizzata dagli studenti dell'Accademia. Poi, per lui, le sole strade praticabili furono le mostre d'Arte Moderna organizzate a Lisbona sull'esempio dei Salons parigini. Nel 1938 le sue tele per la terza Exposição de Arte Moderna furono respinte. Il solo riconoscimento che ebbe, in vita, fu una borsa di studio di due anni concessagli dalla Junta Nacional de Educação nel 1940. Dominguez Alvarez stava allora lavorando a un pannello intitolato Paisagem com Animais. Questo lavoro doveva concludere il suo percorso artistico. Esso rappresentava una sorta di visione globale dell'umanità. Gli animali erano la metafora dell'uomo. Le loro posizioni, la loro vita brutale dominata e regolata dall'istinto di sopravvivenza, rimandava la realtà dell'uomo ingabbiato dall'ipocrisia e stritolato dalla logica spietata dei rapporti sociali.

Centro de Arte Moderna di Lisbona, 1984. E, poi particolarmente aggiornato il catalogo della mostra Almada Dessins, Parigi, Centre Culturel Calouste Gulbankian, 12 ottobre-16 dicembre 1993. Per i rapporti tra i principali esponenti del Modernismo portoghese e i coniugi Delaunay, si veda il bel lavoro di P. Ferreira, Correspondance de quatre artistes portugais. Almada Negreiros, José Pacheco, Souza-Cardoso, Eduardo Viana avec Robert et Sonia Delaunay, Paris, Presses Universitaires de France, 1972. Su Eduardo Viana, si veda la monografia dedicatagli da José-Augusto França, Lisboa, Artis, 1968. Sul Futurismo portoghese e il ruolo di Fernando Pessoa come "critico militante", si veda il saggio di N. Júdice, O Futurismo em Portugal, in Portugal futurista, Lisboa, Contexto Editora, 1990, pp. 1-7. Su questi artisti, e in generale sul clima artistico portoghese fra le due guerre mondiali, si veda ora Modernismo in Portogallo 1910-1940. Arte e società nel tempo di Fernando Pessoa. Firenze, Olschki, 1997 [catalogo della mostra svoltasi a Palazzo Medici Riccardi di Firenze, 23 maggio-20 luglio 1997, a cura dello scrivente]. 
Nel 1942 Candido Dominguez Alvarez morì, stroncato dalla tubercolosi. La sua fortuna critica ebbe inizio dalla retrospettiva organizzata dall'Instituto de Alta Cultura pochi mesi dopo la sua morte. Il comitato scientifico della mostra contava su personalità di prestigio nel Portogallo dell'epoca, come Antonio Ferro, Dordio Gomes e Guilherme Camarinha. Nella sua breve parabola espressiva, la pittura di Candido Dominguez Alvarez si era evoluta dai giovanili paesaggi della Galizia, a una pittura d'irrequieto impianto visionario.

Le scene di vita quotidiana hanno la minaccia incombente di una tragedia che pare stia per compiersi. Ubriachi, sognatori, visionari cominciano a popolare le tele di Dominguez Alvarez negli anni '30. A loro è dato vedere lontano poiché a loro è concesso di vivere al di fuori dei canoni borghesi. Dominguez Alvarez è come il burattinaio di un mondo portoghese provinciale ed eterno, avvolto in una sua dimensione di surrealtà, che ha dei notevoli punti di contatto con il mondo di Strapaese. Ma Dominguez Alvarez, condannato dalla tubercolosi come Scipione, non spinge mai la sua pittura verso una forma, sia pure tenue, d'impegno sociale o politico. La sua pittura è l'omaggio un po' disperato alla vita che si dipana nei piccoli riti, come le bevute di gruppi di amici nelle bettole, come nei momenti di partecipazione collettiva ad un evento.

Un altro soggetto pittorico dell'artista è l'interno dei negozi, nei quali anche la polvere che si deposita sugli oggetti reca un messaggio di mesta poesia. L'affettuosa mestizia che accompagna la pittura di Dominguez Alvarez è talvolta scompaginata da una carica emotiva debordante. È il caso dell' $A u$ toritratto del Centro de Arte Moderna della Fundação Calouste Gulbenkian, che merita di figurare nelle antologiche degli autoritratti del '900. Lo sguardo del pittore, che segue l'astante in ogni suo movimento, e sembra soppesarlo, inquisirlo, è un brano di grande pittura. Fra i quadri più riusciti ed inquietanti di Candido Dominguez Alvarez figura certamente $\mathrm{O}$ Bispo. 
Sorprende che il pittore italiano e il pittore portoghese, accomunati da una morte precoce per la medesima malattia, abbiano dipinto un quadro attraversato da un tormento psicologico di uguale segno e di medesima forza. Ciò che i due testi hanno in comune, è la profondità amara dello sguardo, che scrosta la pellicola invecchiata della pelle e indaga il disfacimento irrimediabile del corpo. A Candido Dominguez Alvarez, pittore degli ubriachi e dei poeti senza spessore corporeo, piegati su sé stessi, incapaci di camminare, portati da un vento impietoso, sembrava che il prelato, sfarinandosi, lasciasse solo il vuoto delle sue dimensioni fisiche. Dal dipinto di Dominguez Alvarez pare di ricavare un silenzioso giudizio negativo sulla spiritualità dell'uomo.

Scipione e Alvarez dipinsero resoconti di sogni, scenari nei quali il significato si palesa solo attraverso il ricorso a codici con i quali ci si avvicina al mondo onirico. Scipione approfondì il proprio legame con la fede. Alvarez ammorbidì il proprio dolore con il recupero di climi chisciotteschi. Dipinse la sua solitudine con i colori forti e pastosi raccolti nelle deambulazioni ebbre per Toledo, Segovia, Caceres; accentuò la sua visione amareggiata in un registro espressivo che forma i suoi paesaggi duri, solidi, irreali, intimamente legati a una tradizione poetica, non solo pittorica, fortemente radicata nei territori di confine fra Spagna e Portogallo. Scipione lavorò in un periodo storico nel quale, sotto la suggestione e la guida di Roberto Longhi, la storia dell'arte italiana veniva letta e assorbita come un immenso deposito di ricchezze formali.

Nel 1930 Longhi curava il catalogo della collezione di artisti spagnoli presenti nella raccolta Contini Bonacossi. Goya e Velazquez venivano indicati agli artisti italiani come termini di paragone e come completamento della tradizione che dall'Italia s'irradiava. Scipione non poteva non tenere conto del lavoro critico di Roberto Longhi, come del resto non ignorò altre fonti critiche, espressioni di un tempo felice 
per la storia dell'arte, forse mai come allora, in Italia, così inserita nel patrimonio culturale condiviso.

Che Scipione, per il tramite del catalogo della Biennale veneziana, possa essere stato una fonte per O Bispo di Dominguez Alvarez è possibile. Scipione e Dominguez Alvarez mostrano un'evidente sintonia nel rovesciare sul ritratto un mondo interiore che pretende diritto di cittadinanza e tende a sovrapporsi al mondo, altrimenti convenzionale, della pittura. L'uomo di Chiesa, investito da ondate emotive d'indicibile violenza, si sgretola e si ricompone. La santità non assurge nei cieli dell'inattingibile lontananza, ma si forgia al fuoco degli istinti, dalla passione, dell'anelito insopprimibile alla vita. 